\title{
Congestion Control in Wireless Sensor Network using HEED Protocol
}

\author{
Meena S. Doibale, G. D. Kurundkar
}

\begin{abstract}
Tens or thousands of sensor nodes are usually deployed in WSN, dispersed in an area with one or more sinks. In addition, several multi-path routing protocols have been proposed to achieve reliability and load balancing. But these protocols limitation is overhead traffic. Thus, congestion is more likely to occur in this situation. HEED (Hybrid, Energy-Efficient, and Distributed) it is a popular distributed clustering protocol in which gives energy and communication costs in a probabilistic manner to elect Cluster Heads (CHs). This paper uses blurred logic and a non-probabilistic approach to CH elections to improve the HEED protocol. To elect CHs, the protocol uses the remaining energy of the node. In contrast, the probabilistic HEED model for the choice of tentative $\mathrm{CH}$ is eliminated by introducing delays for each node, inversely proportional to the residual energy of the node. Provisional CHs are therefore selected on the basis of priority. Here we are performing the comparison of our approach with LEACH (Energy Adaptive Clustering Hierarchy) protocol. Simulation results show that in terms of network lifetime and also cluster formation, our approach performs better than LEACH.
\end{abstract}

Keywords: Sensor networks, clustering, network lifetime, energy efficiency, LEACH, HEED.

\section{INTRODUCTION}

A wireless sensor network (WSN) is a network involving of small-dimension and less-multifaceted devices termed as nodes which can intellect surroundings and collect data from observing arena and connect over wireless links; composed information is progressed, by numerous steps communicating to a sink which can use it in the neighborhood or is associated to other systems [1]. A node typically contains of 4 subsystems: detecting unit, processing unit, communiqué unit as well as power unit. Nodes are installed in a sensor arena [2]. The BS is a dominant node and information detected by system is transmitted back to BS [3]. The BS may transfer with extra sensor nodes. HEED (Hybrid Energy Efficient Distributed) is clustering protocol with energy efficiency. It uses remaining energy as principal factor, node degree and distance to neighbors as next factors. It prolongs the uncomplicated structure of LEACH protocol. Clustering is divided into multiple steps. In each single step, sensor nodes which are not enclosed by any cluster; it generally doubles the possibility of becoming a cluster head

Revised Manuscript Received on July 22, 2019.

* Correspondence Author

Meena S. Doibale, Research Scholar, Department of Computational Sciences, S.R.T.M.University, Nanded, Maharashtra, India.

Email: meena.doibale@gmail.com

Dr. G.D. Kurundkar, Shri Guru Buddhiswami Mahavidyalaya, Purna(Jn.),

Dist. Parbhani, Maharashtra, India.

Email: gajanan.kurundkar@gmail.com
(CH). A HEED protocol [4] shown in Figure 1 shows transmission of data takes place

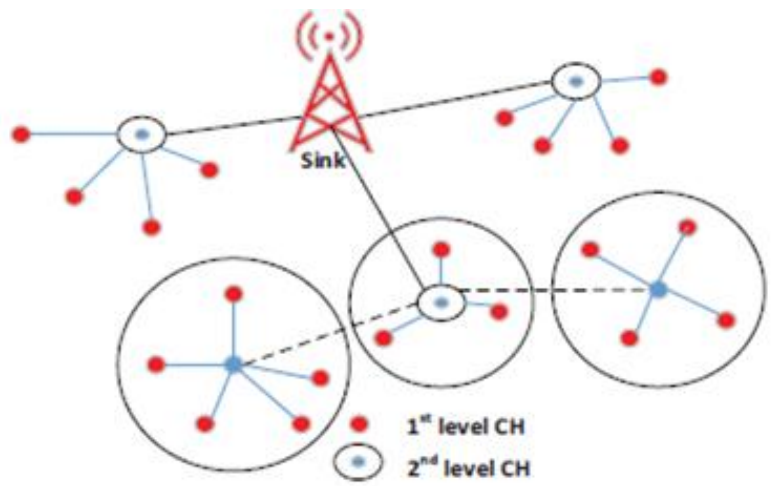

Fig.1 Data routing in HEED protocol

It cannot assurance optimum selected set of CHs. Heterogeneous -HEED (H-HEED) [5] is the adapted type of HEED protocol in order of non-uniformity. The $\mathrm{CH}$ is nominated by some portion of remaining energy to supreme energy influenced by the nodes. It communicate directly and unalike energy smoothed systems have been generated [6][7].

This work is organized as follows. Section 2, present the system model and energy model. Section 3 presents a proposed system algorithm which compare with existing system. In which we have described Heed protocol in brief and also present LEACH algorithm. Section 4 presents a brief description result. Section 5, we conclude our work

\section{SYSTEM MODEL}

\section{A. Energy Model}

In this paper, the same wireless communication energy consumption model is used in literature. In this model, the energy consumption of the data transmission by the wireless communication module is mainly in the transmission circuit and the power amplifier circuit, and the energy consumption of received data mainly in the receiving circuit [9]. In order to ensure reasonable signal to noise ratio, the node sends data energy consumption as follows: 


$$
\begin{gathered}
E(d, k)=\left\{\begin{array}{l}
E_{\text {elec }} \times k+E_{\text {ss }} d^{2} \\
E_{\text {elec }} \times k+E_{\text {amp }} d^{2}
\end{array}\right. \\
d<d_{0}, d \geq d_{0}
\end{gathered}
$$

Among them: $\mathrm{k}$ is the binary number of data to send, $\mathrm{d}$ is the transmission distance; Eelec is the RF energy consumption coefficient, Efs and Eamp are the energy dissipation coefficients of the power amplifying circuit under different channel propagation models. When the transmission distance is less than the threshold value, the power amplification loss adopts the free space model. When the transmission distance is greater than or equal to the threshold value $\mathrm{d} 0$, the multi-path attenuation model is adopted [9]. The energy consumption of the node receiving data is

$$
E_{R X}(k)=E_{\text {elec }} \times k
$$

\section{Network Model}

Similar to HEED, we assume the following sensor network properties:

- The nodes can change the amount of transmit power using power control

- Links are symmetrical, i.e. with the same transmission power, two nodes v1 and v2 can communicate

- Nodes are constrained by energy. The distance can be measured based on the wireless radio signal power

- Node failures are primarily due to the depletion of energy.

\section{Proposed System}

We have implemented HEED (Hybrid, Energy-Efficient, and Distributed) algorithm with respect to various input data sets, we have tested it by using varying size of node, data transmission limits and input data size. All of the above implantation is compared with LEACH (Energy Adaptive Clustering Hierarchy) algorithm. Both the algorithms are described as below:

\section{HEED}

WSNs include a huge number of nodes fluctuating in hundreds or may be thousands. The 1st stage includes in this segment is the scheming of nodes in a $100 * 100 \mathrm{~m}$ square region. 300 sensor nodes with the BS are positioned. At first, energy of each sensor nodes is assigned as 0.5 joule. For the $\mathrm{CH}$ choice amongst ' $\mathrm{n}$ ' numbers of sensor nodes average distance of every single nodes with extra nodes from the similar cluster is achieved by equation (1) that is given-

$$
\phi(n)=\frac{d_{1}+d_{2}+\ldots \ldots . d_{n i 1}+d_{n}}{n}
$$

Where di is the distance among any two neighboring sensor node that is computed by Euclidian distance formula given in equation (2)

$$
\left.d_{i}=\sqrt{\left(x_{i}-x_{j}\right)^{2}+\left(y_{i}\right.}-y_{j}\right)^{2}
$$

Where $\left(x_{i}-x_{j}\right)$ and $\left(y_{i}-y_{j}\right)$ are the co-ordinates of the sensor nodes.

So possibility of any sensor node to become $\mathrm{CH}$ is given by equation (3)

$$
C H_{\text {prob }}=C_{\text {prob }} \mid * \frac{E_{\text {residual }}}{E_{\text {max }}}
$$

Where

$$
\begin{aligned}
& C_{\text {prob }} \text { : Initial probability } \\
& E_{\text {residual }} \text { : Residual energy } \\
& E_{\max } \text { : Maximum energy of nodes }
\end{aligned}
$$

We have considered 300 sensor nodes that are distributed in $100 * 100 \mathrm{~m}$ square area. The validation condition applied to the network is as bellow:

- Sensor nodes in the system are immobile.

- Sensor nodes positions are uninformed i.e. it is not prepared via the GPS accomplished antenna.

- Sensor nodes have parallel processing and communiqué abilities and identical consequence.

- Sensor nodes are neglected after placement

$\mathrm{CH}$ selection is mainly by remaining energy of every single sensor node. As energy spent per bit for detecting, processing and communiqué is characteristically identified and therefore remaining energy may be expected. Intra-cluster communique budget is well thought-out as the secondary factor to break the links. A link means that a sensor node might tumble inside the range of more than one $\mathrm{CH}$. When multiple $\mathrm{CHs}$, the individual with lower intra-cluster communication are preferred.

In our proposed protocol, supposing system to be dissimilar in nature, where total $\mathrm{m} \%$ of advanced node have additional energy factor in this one while equating with normal nodes. Supposing every single sensor node has initial energy. Energy of advanced sensor node in recommended protocol is $E_{0}(1+\beta)$. Now construction of total energy of projected network is computed by equation (4)

$$
N E_{0}(1+m \beta)=n m E_{0}(1+\beta)+N(1-m) E_{0}
$$

As an outcome over-all energy is amplified by factor $(1+\beta m)$ times. According to possibility of normal node and advanced node we heightened election technique with residual energy of specific nodes. Normal member node probability function is defined by equation (5)

$$
P_{\text {normal }}=\frac{P_{\text {opt }}}{1+\beta m} \times \frac{E_{\text {residual }}}{E_{0}}
$$


Where $P_{o p t}$ optimal percentage of $\mathrm{CH}$ and $E_{o}$ is preliminary energy of sensor nodes. Likewise advanced sensor node probability function is defined by equation (6)

$$
P_{\text {advance }}=\frac{P_{o p t}}{1+\beta m} \times \frac{E_{\text {residual }}}{E_{o}} \times(1+\beta)
$$

Selection of $\mathrm{CH}$ is defined by equation (7)

$$
T(n)=\left\{\frac{P}{1-P(r \bmod 1 / p)}_{\text {otherwise }}^{n \in G}\right.
$$

Where

- $\mathrm{P}$ is proportion of choosing $\mathrm{CHs}$.

- $\mathrm{r}$ is present round.

- $\mathrm{G}$ is group of all the sensor nodes which are not in $\mathrm{CHs}$ in $1 / \mathrm{P}$ rounds

In every single round, node produces random number belonging to 0 and 1 . If generated random number is lesser than $\mathrm{T}(\mathrm{n})$ then selected as $\mathrm{CH}$ otherwise not.

\section{The Algorithm of LEACH}

LEACH (Low Energy Adaptive Clustering Hierarchy) was the WSN routing protocol which is sub-cluster-style. Most of the subsequent sub-cluster routing protocols are developed on the basis of LEACH. LEACH conserves energy because it uses data compression techniques and sub-cluster dynamic routing mechanics. It randomly select a node as cluster head node and balances the load of the network to control the rapid loss of cluster head nodes [10].LEACH implements a cyclic process. The cycle is divided into clusters of the establishment phase and stable data communication phase [11].

The cluster head selection process of LEACH is as follows: a random number generated by nodes is between $0-1$,

if it founds that this number is less than the thresholds $\mathrm{T}$ (n), then it publish a notice that they are cluster head messages [10]. The probability of node selection is T (n); the probability of cluster head node selection will increases. $T$ (n) can be expressed as:

$$
T(N)=\left\{{\frac{P}{1-p \times\left[r \bmod _{0}(1 / p)\right]}}_{n \notin G}^{n \in G}\right.
$$

$\mathrm{P}$ denotes cluster head in of all nodes, $\mathrm{r}$ denotes the number of selection rounds, $\mathrm{r}$ mod $(1 / \mathrm{P})$ denotes the number of cluster head nodes selected in a cycle, $\mathrm{G}$ denotes node set which is not selected as cluster head.

\section{The Cluster Head Selection}

Because of in adequacy of the formula T (n) in LEACH, the formula of $T(n)$ is upgraded.

$$
T(N)=\frac{P}{1-p \times\left[r \bmod _{0}(1 / p)\right]} \frac{E_{n_{-} \text {current }}}{E_{n_{-} \max }}
$$

$E_{n_{-} \text {current }}$ denotes nodes current energy, $E_{n_{-} \max }$ denotes initial energy. The (9) formula can be enhance, so a node is selected as cluster head whose energy consumption is lower. [10]. Exploratory out comes gives that the life span of network is improved by $20 \%$ by the node selection using LEACH. Per contra, formula (2), has an error for this upgrade. As the network is running for a long time duration, the current nodes energy become low, then the threshold $\mathrm{T}(\mathrm{n})$ will be less, it reduces the all nodes probability to be selected as a cluster, one and all the chosen cluster head reduce, eventually network energy utilization is not equitable, the life span of network get lesser. Remarkably T (n) is improved. rs denotes the chosen cluster head rounds [12]. As cluster head get chosen, $r$ is resets to 0 .

\section{The Cluster Head Competition}

After acquiring the network parameters, cluster head is generated by rotation approach [14]. In order to balance the node energy consumption, the radius of all nodes is set by non-linear manner and the hops of the node to the sink. The area $S$ (h) is the h-hop distance from sink node, Rc (h) is a radius of the region of the cluster head.

$$
n\left(h_{\max }\right)=S\left(h_{\max }\right) /\left(\pi\left(R_{s} / s\right)^{2}\right)
$$

Then the region can be formed the cluster which is not less than $S\left(h_{\max }\right) / \pi R_{c}^{2}(h)$

So set $\mathrm{n}(\mathrm{h})$ to the number of cluster with hop $\mathrm{h}$. When $\mathrm{h}=\mathrm{h}_{\max }$, competition radius of the node is $\mathrm{R}_{\mathrm{s}} / 2$. With the node $\mathrm{V}_{\mathrm{i}}$ with the hop $\mathrm{h}_{\mathrm{i}}$, if $\mathrm{V}_{\mathrm{i}}$ becomes the cluster head, each cluster head moves data from the hierarchy to a higher hierarchy,

$$
\text { Vi sent } m_{i}=h_{\max }-h_{i}+1
$$

By equation (11), the more cluster head close to the sink need to forward more data. If the cluster head close to the region of sink, it will help balance the cluster head energy consumption.

$$
n(h)=n\left(h_{\max }\right) f(h)
$$

Set $\mathrm{f}(\mathrm{h})$ is decreasing function with $\mathrm{h}, \mathrm{f}(\mathrm{h}) \geq 1, \mathrm{f}(1)=\mathrm{a}$, a $\geq 1$ a is constant variable, ( ) $1 \mathrm{fh}_{\max }=\mathrm{n}(\mathrm{h})$ is changed with . The rate of change

$$
\begin{gathered}
\Delta n(h)=n\left(h_{\max }\right) \Delta f(h)=(-\beta / h) n\left(h_{\max }\right) \\
n(h)=n\left(h_{\max }\right)(-\beta 1 n(h)+C)
\end{gathered}
$$

$\mathrm{C}$ is undetermined coefficient. 


$$
n(h)=\left((1-a) \frac{1 n(h)}{1 n\left(h_{\max }\right)}+a\right) n\left(h_{\max }\right)
$$

The area of the circular area with sink as the center, the distribution of the ring area can be broadly expressed as:

$$
S(h)=(2 h-1) \pi\left(R_{s} / 2\right)^{2}
$$

From formula (10) and (16), it can deduce

$$
n\left(h_{\max }\right)=2 h_{\max }-1
$$

From formula (15), (16) and (17), the radius of the competition with h hop is

$$
R_{c}(h)=\frac{R_{s}}{2} \sqrt{\frac{2 h-1}{\left.\left.2 h_{\max }-1\right)(111-a) \frac{1 n(h)}{1 n\left(h_{\text {max }}\right)}+a\right)}}
$$

Leach Algorithm having more energy consumption than HEED algorithm. From above algorithm it is clear that leach having more delay time and also require more computational time.

\section{Result Discussion}

We have proposed an HEED algorithm analysis of energy consumption and end to end delay analysis. Here we get various result which are then compared with LEACH protocol. We have discuss result as bellow

\section{Energy Consumption Analysis}

This parameter is to be used to check out the lifetime of the network. If the energy consumption is higher, that node will be go out of power soon and the network lifetime will be decreased which will have adverse effect on the network. Energy consumption here shows the total energy consumption by the nodes that are intermediate nodes from source to destination nodes. Energy Consumption is essential parameter that can show the capacity of nodes that participates in the network.

In Table I, the author analyze that the energy consumption of our proposed work is very less. In the previous work the energy consumption is high i.e. it consumed upto $80 \%$ of total energy of the network and this proposed work consumed $20 \%$ of the network. This percentage is also less as DDR technique is apply in clustering and it is always skipped in previous work.

Table- I: Energy Comparison in Joule (J) for 300 node simulation

\begin{tabular}{|l|c|c|l|}
\hline $\begin{array}{c}\text { Energy } \\
\text { Consumption }\end{array}$ & $\begin{array}{c}\text { LEA } \\
\text { CH }\end{array}$ & $\begin{array}{c}\text { HE } \\
\text { ED }\end{array}$ & Difference \\
\hline Minimum & 2 & 0 & 2 \\
\hline Average & 11 & 5 & 6 \\
\hline Maximum & 18 & 8 & 10 \\
\hline
\end{tabular}
the previous work. number of alive nodes.

The delay is the important factor to calculus performance of the network. Speaking about delay, if the arrival of first packet could not be found by the source node and node observed that the second packet arrived after the first packet. This is the defining way of delay. This factor is calculating at the MAC and Network layer or between them. It is also possible, "more the delay later the packet received", resultant poor performance of the network. In Table II, the author measure the delay of the previous / proposed work and analyzed that the delay in the proposed work is less and that's why the consumption of the energy is also less. If the author analyze the difference from the table then the maximum delay of the previous work is high i.e. $30 \mathrm{~ms}$ and maximum delay in proposed work is $18 \mathrm{~ms}$ which is less as compare with

Table-II: Delay Comparison

\begin{tabular}{|l|l|l|l|}
\hline Delay(ms) & $\begin{array}{l}\text { LEAC } \\
\text { H }\end{array}$ & HEED & Difference \\
\hline Minimum & 2 & 0 & 2 \\
\hline Average & 16 & 7 & 9 \\
\hline Maximum & 30 & 16 & 19 \\
\hline
\end{tabular}

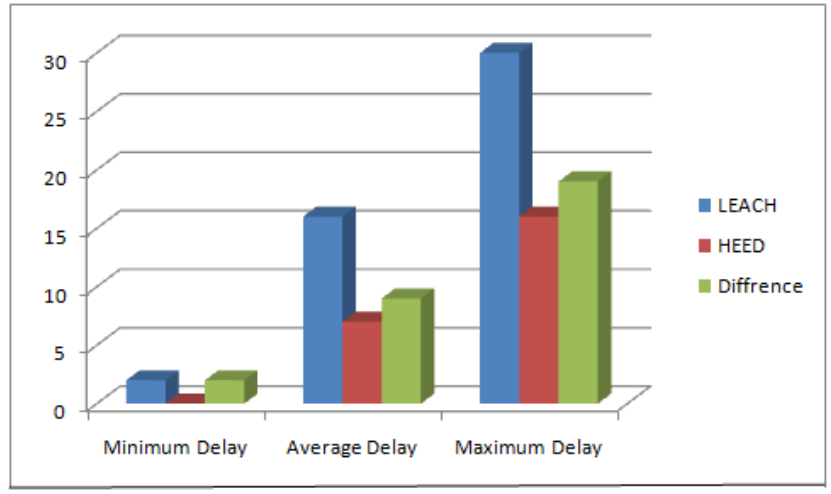

Fig. 4 Delay time of WSN

HEED protocol gives better performance than LEACH. Proposed HEED outperforming well compared to the existing variant of LEACH protocol in terms of dissipated energy, first node die with respect to cluster radius and 


\section{Conclusion}

In this paper, we compare two different protocol, LEACH and HEED. We present protocol for WSN for energy efficiency and distributed clustering. We proceed towards applications that require scalability, prolonged network lifespan and nodes are dispersed in a large spacious field. We consider a quasi-stationary networks in which nodes are location-unaware and have equal significance. Most valuable feature in this approach is the nodes must support variable transmission power. On this assumption, we presented the HEED protocol, where Cluster Head $(\mathrm{CH})$ selection process ends after a constant number of iterations. It does not depend on the network diameter. Non probabilistic approach is performed by assigning "delay time", inversely proportional to node's energy, to each node. By which decrease delay time and also reduce the congestion problem. The HEED is evaluated by comparing it with LEACH by using MATLAB. Hence we conclude that HEED protocol performance is better than LEACH protocol.

\section{REFERENCES}

1. N. A. Pantazis, S. A. Nikolidakis, and D. D. Vergados,

"Energy-Efficient Routing Protocols in Wireless Sensor Networks: A Survey," IEEE Commun. Surv. Tutorials, 2 ed., vol. 15, pp. 551-591, 2013.

2. P. Huang, L. Xiao, S. Soltani, M. W. Mutka, and N. Xi, "The evolution of MAC protocols in wireless sensor networks: A survey," IEEE Commun. Surv. Tutorials, 1 ed., vol. 15, pp. 101-120, 2013.

3. J. Kulik, W. Heinzelman, and H. Balakrishnan,

"Negotiation-basedprotocols for disseminating information in wireless sensor networks," Wirel. Networks, vol. 8, pp. 169-185, 2002

4. C. H. Lin and M. J. Tsai, "A comment on 'HEED: A Hybrid, Energy-Efficient, Distributed clustering approach for ad hoc sensor networks," IEEE Transactions on Mobile Computing, vol. 5, no. 10. pp. 1471-1472, 2006.

5. S. Chand, S. Singh, and B. Kumar, "Heterogeneous HEED protocol for wireless sensor networks," Wirel. Pers. Commun., 3 ed., vol. 77, pp. 2117-2139, 2014.

6. L. Qing, Q. Zhu, and M. Wang, "Design of a distributed energy-efficient clustering algorithm for heterogeneous wireless sensor networks," Comput. Commun., 12 ed., vol. 29, pp. 2230-2237, 2006.

7. R. Q. Hu and Y. Qian, "An energy efficient and spectrum efficient wireless heterogeneous network framework for 5G systems," IEEE Commun. Mag., 4 ed., vol. 52, pp. 94-101, 2014.M. Young, The Techincal Writers Handbook. Mill Valley, CA: University Science, 1989.

8. Mohammed Omari, et al., "Simulation, Comparison and Analysis of Wireless Sensor Networks Protocols: LEACH, LEACH-C, LEACH-1R, and HEED," IEEE, 2015.

9. Jiwei Huang, et al., "Design and Implementation of Multi - hops Wireless Sensor Network Routing

10. Protocol Based on HEED," 17th IEEE International Conference on Communication Technology, pp. 598-6011, 2017.

11. Gitanshu, et al., "Energy Optimization of Hybrid Based H-HEED Protocol in Inter-Cluster Based Networks," IEEE, 2016.

12. Lejiang Guo, et al., "Cluster-based Algorithm for Energy-efficient Routing in Wireless Sensor Networks," 2010 International Forum on Information Technology and Applications, pp. 11101-1103, 2010.

13. Divya.P, et al., "Comparison of GSTEB, HEED and PEGASIS Protocols," IEEE, pp. 1935-1939, 2016.

Mahdi Lotfinezhad, et al., "Effect of Partially Correlated Data on Clustering in Wireless Sensor Networks,” IEEE, pp. 172-181, 2004.

14. Seema Rani Gadai, et al., "Comparison and Analysis between Distinguish Clustering Protocols of Wireless Sensor Networks (A Survey)," International Journal of Computer Applications, pp.22-27, 2016.

15. F. S. Younis O., "A hybrid, energy-efficient, distributed clustering approach for ad hoc sensor networks," IEEE Trans. Mob. Comput., vol. 3 , no. 4, pp. 366-379, 2004.

16. H. Lin, L. Wang, and R. Kong, "Energy Efficient Clustering Protocol for Large-Scale Sensor Networks," IEEE Sens. J., 12 ed., vol. 15, pp. 7150-7160, 2015.
17. E. Ever, R. Luchmun, L. Mostarda, A. Navarra, and P. Shah, "UHEED - an unequal clustering algorithm for wireless sensor networks," Sensornets 2012, 2012.

18. C. Li, M. Ye, G. Chen, and J. Wu, “An energy-efficient unequal clustering mechanism for wireless sensor networks," in 2nd IEEE International Conference on Mobile Ad-hoc and Sensor Systems, MASS 2005, 2005, vol. 2005, pp. 597-604.

19. H. Bagci and A. Yazici, "An energy aware fuzzy approach to unequal clustering in wireless sensor networks," Appl. Soft Comput. J., 4 ed., vol. 13, pp. 1741-1749, 2013.

20. R. Gagliardi, "RUHEED- Rotated Unequal Clustering Algorithm For Wireless Sensor Networks," Procedia Comput. Sci., vol. 57, April 2015, pp. 660-669, 2015.

21. Z. U. Nueraili Aierken, Roberto Gagliardi, Leonardo Mostarda, "RUHEED-Rotated Unequal Clustering Algorithm For Wireless Sensor Networks," in Internation Conference on Advanced Information Networking and Applications Workshops, 2015, pp. 170-174.

22. ] Z. Ullah, L. Mostarda, R. Gagliardi, D. Cacciagrano, and F. Corradini, "A comparison of HEED based clustering algorithms - Introducing ER-HEED," in Proceedings - International Conference on Advanced Information Networking and Applications, AINA, 2016, vol. 2016-May, pp. 339-345

\section{AUTHORS PROFILE}

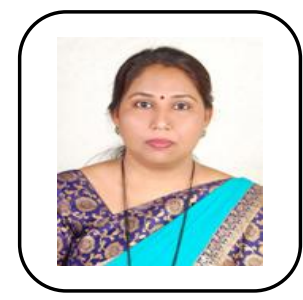

First Author Doibale Meena Shyamrao is pursuing her Ph.D. in School of computational Sciences, S.R.T.M.U. Nanded. Maharashtra, India. She has more than 14 years of experience in teaching field. Her area of interest in "Congestion Control in Computer networks".

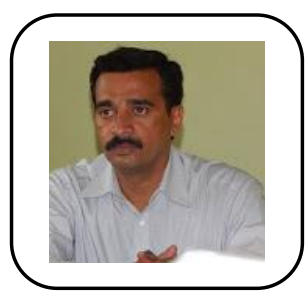

Second Author Dr. Kurundkar Gajanan Dattatray is working as Assistant Professor at Department of Computer Science, Shri Guru Buddhiswami Mahavidyalaya, Dist. Parbhani,

Maharashtra, India. His area of interest is Computer Networks. He has published 4 Books on Computer Networks. He is Life Member of Indian Science Congress Association, Kolkata. Member of (IAENG) International Association of Engineers

Member. He has more than 16 years' experience in teaching field. 